\title{
Aproximación Psicosocial y de Género al Proyecto Migratorio de Mujeres
}

\section{A Psychosocial and Gender Approach to the Migratory Project of Women}

\begin{abstract}
Victoria Zarco Martín*, Pilar Díaz Conde, Mª Angustias Martín Quirós, Carmen Ardid Muñoz y Nuria Rodríguez Salas ${ }^{1}$

"Los estudios sobre el nuevo problema social de la inmigración en España y sobre las políticas públicas que intentan regularlo adolecen con frecuencia de unilateralidad. Un hecho social como el proceso migratorio exige un acercamiento complejo" (Raya, 1999).
\end{abstract}

\begin{abstract}
Resumen
A pesar de la presencia cada vez más significativa de mujeres inmigrantes ${ }^{2}$ en España, se observa en la investigación sobre este tema dos sesgos importantes que impiden una correcta explicación del fenómeno migratorio y una adecuada intervención. En primer lugar, la mayoría de los estudios realizados están relacionados con el proyecto migratorio masculino. En segundo lugar, la importancia que adquieren los aspectos socioeconómicos y políticos en el análisis de los fenómenos migratorios ha hecho que haya sido la perspectiva macrosocial la que ha dominado esta área de estudio.

En este artículo se intenta profundizar en el estudio de la mujer inmigrante desde una perspectiva psicosocial y de género, haciendo especial hincapié en las principales variables psicosociales que definen su proyecto migratorio y ubicando dicho proyecto en un enfoque de género, pues no cabe duda que éste se encuentra fuertemente modulado o mediatizado por una serie de factores que proceden directamente de su condición de mujer.
\end{abstract}

Asimismo, en el presente artículo se esbozan algunas cuestiones que se podrían tener en cuenta en las futuras líneas de investigación para lograr una adecuada comprensión del fenómeno migratorio femenino y para dirigir la intervención hacia el incremento de la satisfacción, la calidad de vida y el bienestar de la mujer inmigrante.

Palabras clave: Proyecto migratorio, mujer, aproximación psicosocial, enfoque de género.

* Academica, Universidad de Granada. España.vzarco@ugr.es

1 Las autoras de este artículo participan en la actualidad en un proyecto de investigación, subvencionado por el Instituto de la Mujer, y titulado "Aproximación psicosocial y de género al proyecto migratorio de las mujeres en Andalucia".

2 A lo largo del presente artículo hemos adoptado el término de "mujer inmigrante", ya que la reflexión teórica y metodológica que se realiza en estas páginas se centra en la mujer extranjera que viene a España para desarrollar su proyecto migratorio.a 


\begin{abstract}
Despite a recent increase in both immigration to Spain, and studies on immigrants, research concerning women immigrants has two important biases that prevent correct explanations of the migration, as well as limiting suitable interventions. Firstly, most studies relate to the masculine migratory project. Secondly, the dominance of social, economic and political aspects in the analysis of migratory phenomena means that the macrosocial perspective has been the prevailing focus in the study of this topic.

In this paper we adopt a psychosocial and gender based approach to analysis of the situation of women immigrants. We emphasise the main psychosocial variables that define their migratory project, since it is clear that they are strongly influenced by different factors, linked directly with their women's condition.

In addition, in this paper, we outline some questions that should be considered in future research in order to achieve a correct understanding of the feminine migratory phenomenon is to be reached, and so that future intervention may increase women immigrant's satisfaction, quality of life and welfare.
\end{abstract}

Keywords: migratory project, woman, psychosocial approach, gender approach.

\section{La importancia del proceso migratorio en España}

Una de las características fundamentales que definen a las sociedades industrializadas de finales del siglo XX es la gran variedad y la rapidez de los cambios (tecnológicos, económicos, políticos, sociales y culturales) que se han producido en ellas en las últimas décadas y que, aún hoy en día, se siguen produciendo. Estos cambios han propiciado, entre otros acontecimientos, el establecimiento de nuevas relaciones a escala mundial y una globalización de la economía y han conllevado, como consecuencia, la aparición de nuevos fenómenos y problemas sociales que requieren una comprensión y una solución urgente por parte de los investigadores e instituciones competentes.

No cabe duda que de estos fenómenos es el proceso migratorio internacional que se da en la actualidad como uno de los más representativos. En este sentido, es un hecho frecuentemente constatado que Espa- ña ha dejado de ser un país de emigración para convertirse en un país receptor de inmigrantes. Más concretamente, podríamos afirmar que es a mediados de los años ochenta cuando empieza a apreciarse una llegada significativa de inmigrantes, procedentes tanto de Europa como de otras zonas en vías de desarrollo, a nuestro país, si bien ha sido el primer gran proceso de regularización de inmigrantes llevado a cabo en 1991 el que ha consolidado a España como un país de inmigración.

Entre las causas (Martínez, 1999; López de Lera, 1995; Raya, 1999) de esta transformación se encuentra fundamentalmente la incorporación de España a la Unión Europea, circunstancia que le ha permitido reafirmarse como país desarrollado económicamente, relativamente rico y, además, política y socialmente estable. De otro lado, la peculiar posición geográfica de España la convierte en trampolín de numerosos inmigrantes que se dirigen a otros destinos de la U.E. Asimismo, factores como el desarrollo de las políticas y los servicios del 
estado de bienestar español, el desplazamiento de población inmigrante hacia otros países por el cierre de fronteras de aquellos que tradicionalmente recibían un mayor flujo de proyectos migratorios y el nuevo papel de la economía española en la división internacional del trabajo, contribuyen a esta transformación en nuestro país.

Por último, no podemos olvidar las peculiaridades de nuestro mercado de trabajo, definido por dos características fundamentales para la comprensión del fenómeno migratorio actual: su carácter dual y segmentado. Desde este último punto de vista, se observa que la mayor parte de la población inmigrante se incorpora en la denominada "economía sumergida", convirtiéndose así ésta en un factor de movilidad y de flexibilidad del mercado de trabajo español (López y Ramírez, 1997). Igualmente, y muy relacionado con lo anterior, existen en este campo informal de la economía unos sectores considerados "duros" que, al ser progresivamente abandonados por la mano de obra nacional, son, precisamente, los que se reservan para los inmigrantes. En este sentido, se expresan algunos autores cuando afirman que el servicio doméstico, junto con la agricultura y la construcción son sectores que se han reforzado y han podido remontar su crisis gracias a la inmigración extranjera.

Por otra parte, si hacemos un breve análisis de las causas que impulsan a la emigración, existe cierta unanimidad en expresar que son las condiciones de inestabilidad económica del país de origen y, por tanto, la falta de oportunidades que ofrece este entorno a los individuos para la satisfacción de sus necesidades y expectativas, las principales responsables de la huida de las personas hacia otros países en busca de la mejora de su posición social y económica. No obstante, es preciso señalar que aunque el condicionamiento económico es uno de los factores fundamentales para explicar el proyecto migratorio de los individuos, tam- bién es cierto que en la actualidad existe una variedad de motivos ajenos a la pobreza que habría que analizar.

Así pues, observamos como el fenómeno migratorio internacional y, más concretamente, la creciente presencia de población inmigrante en España está directamente relacionada con la situación económica global de los países de procedencia de los trabajadores extranjeros y con una serie de características actuales del Estado Español que lo convierten en un país de un gran atractivo para la realización del proyecto migratorio, entre las que se encuentran fundamentalmente las derivadas del propio mercado laboral español.

Es en este marco interpretativo del fenómeno migratorio español donde vamos a ubicar el análisis de la mujer inmigrante ya que, como veremos posteriormente, la presencia cada vez más significativa de ésta en nuestro país se debe a una serie de facilitadores económicos y sociales, procedentes tanto del país de origen como de destino, que la impulsan a iniciar su proyecto migratorio. Asimismo, en este análisis nos acogeremos a una definición amplia del concepto de inmigración desde la que se nos permita incluir en este proceso a todas aquellas mujeres que por diversos motivos (políticos, económicos, familiares, religiosos, étnicos o profesionales) se desplazan a España con el propósito de mejorar su situación.

\section{Hacia una progresiva feminización de la población inmigrante}

Si atendemos a la composición actual de la población inmigrante internacional, incluida la española, podemos decir que ésta presenta con respecto a la que existía tradicionalmente tres características básicas: es más femenina, más familiar y más estable y permanente. Son numerosos los investi- 
gadores que ratifican la progresiva feminización del proceso migratorio, hecho que se traduce en una movilidad femenina comparable a la del varón y, por tanto, en una percepción social cada vez más frecuente de la mujer como la principal protagonista del proyecto migratorio. Desde este punto de vista, se estima que en España más de un tercio de los trabajadores inmigrantes son mujeres.

Podríamos hablar de tres posibles explicaciones a este proceso de feminización. En primer lugar, el cambio en la composición demográfica de los nuevos movimientos migratorios puede ser debido a una reconsideración de los roles tradicionales y de la posición social de la mujer emigrante en su sociedad de origen, tendiendo a una mayor emancipación femenina e igualdad entre los sexos. Este, por ejemplo, es el caso de la actual inmigración marroquí a España, donde las mujeres inmigrantes están asumiendo un papel económico activo, pero inédito hasta el momento en los países árabes, ayudando así al reconocimiento explícito de la figura de la mujer como emigrante $\mathrm{y}$, por tanto, estimulando la movilidad femenina (López y Ramírez, 1997).

En segundo lugar, no podemos olvidar que la pobreza afecta más a las mujeres que a los hombres ("feminización de la pobre$\mathrm{za}^{\prime \prime)}$, siendo las mujeres rurales de los países más desfavorecidos uno de los grupos más vulnerables a la crisis económica y, por ello, más predispuesto a la emigración (Gregorio, 1994).

Por último, en España, como ya hemos aludido anteriormente, existe una segmentación étnica y sexual del mercado de trabajo que favorece una demanda creciente de mano de obra femenina extranjera para sustituir a las mujeres de nacionalidad española en el servicio doméstico, sector que entró en crisis desde el fin de la emigración interior a finales de la década de los sesen- ta. Este último factor explicativo queda suficientemente ilustrado si recordamos que en el año 1998 del contingente de autorizaciones para el empleo de trabajadores extranjeros no comunitarios, fijado en un total de 28.000, 14.205 correspondieron al servicio doméstico (Cardona, 1999).

\section{Necesidad de un enfoque psicosocial y de género en el estudio del proceso mi- gratorio en España}

A pesar de la presencia significativa de mujeres inmigrantes en España, observamos en la literatura e investigación sobre este tema dos sesgos importantes que impiden una correcta explicación del fenómeno migratorio, así como una adecuada intervención. En primer lugar, la mayoría de los estudios realizados están relacionados con el proyecto migratorio masculino. Esta "invisibilidad" de la emigración femenina es fruto de varios factores entre los que destacan la existencia cada vez más frecuente de una reagrupación familiar ilegal, la inserción en los sectores más sumergidos de la economía española, pero, también, y no menos importante, la posición subalterna de la mujer tanto en las sociedades emisoras como en las receptoras (Martín, 1999).

Se hace patente, pues, la necesidad de un enfoque de género desde el que se pueda explicar este silenciamiento y analizar más exhaustivamente las variables que dotan de peculiaridad al proyecto migratorio de las mujeres y el grado en que dicho proyecto se encuentra mediatizado por variables procedentes de su condición de mujer, $\mathrm{y}$, más exactamente, de su proceso diferencial de socialización.

Un segundo sesgo alude a la importancia que adquieren los aspectos socioeconómicos y políticos en el análisis de los fenómenos migratorios, siendo la perspectiva macrosocial la que ha dominado esta 
área de estudio. No obstante, es posible y, además, necesario un acercamiento multidisciplinar, donde tenga cabida una reflexión de carácter más "micro" o psicosocial, pues un mayor y mejor conocimiento del entorno microsocial de la mujer inmigrante ayudaría a superar las barreras que limitan su integración laboral satisfactoria en el país de destino, así como a aprovechar las ventajas y oportunidades que su perfil psicosocial les ofrece.

Desde este último planteamiento, no cabe duda que en el caso de la mujer inmigrante no sólo podemos hablar de variables macrosociales, procedentes del país de origen y de destino, como determinantes de su situación, sino que, además, su proyecto es susceptible y debe ser analizado desde un enfoque psicosocial, investigando sus motivos para emigrar, los recursos personales, sociales y económicos de los que dispone para hacer frente a su nueva situación, las razones para elegir el país de destino, sus estrategias de búsqueda de empleo, sus actitudes hacia la integración, el grado de satisfacción y de bienestar, etc.

Así pues, creemos que es necesario continuar la línea de investigación desde la que se intenta profundizar en el estudio de la mujer inmigrante desde una perspectiva psicosocial y de género, no sólo por su carácter menos sesgado $\mathrm{y}$, por tanto, más realista en la explicación de la situación de estas mujeres, sino también porque pensamos que es una vía valiosa y útil de obtener información que permita a las instituciones competentes comprender e intervenir en lo que consideramos un doble problema: ser mujer $\mathrm{y}$, además, inmigrante.

Asimismo, nuestra postura viene ratificada por algunas investigaciones desde las que se constata que la mujer inmigrante posee una problemática específica que es necesario abordar con una metodología y con unas técnicas diferenciadas, pues ésta se inserta en procesos de trabajo diferentes a los de los hombres, posee actitudes y valores distintos del varón con respecto a la experiencia migratoria, así como establece y percibe de manera diferente las relaciones interétnicas. Es decir, el hecho de ser mujer e inmigrante da lugar a una serie de situaciones que deben ser analizadas en su especificidad, hecho que ayudará a paliar la invisibilidad de las mujeres en el proceso migratorio internacional (Martín, 1999).

\section{Características del proceso migratorio femenino desde una perspectiva de género}

A través del análisis de algunos estudios e investigaciones realizadas (Hita, 1999; Cardona, 1999; Prado y cols, 1995, 1997; Gregorio, 1994, 1997, 1998; Gregorio y Franzé, 1999; Gailly y Driss, 1997) se pueden extraer algunas de las razones que sustentan la necesidad de realizar una aproximación de género al estudio de las migraciones. Veamos brevemente las más significativas.

En primer lugar, observamos que las mujeres presentan cierta diversidad con respecto a la concepción de su proyecto migratorio. En este sentido, podemos hablar de tres tipos de mujeres inmigrantes: las inmigrantes "pasivas", que llegan al país de destino para unirse al esposo trabajador y, por ello, muy ligadas al proceso de reagrupación familiar; las inmigrantes "activas", que emigran solas y que posteriormente inician el proceso de reagrupación familiar. Por último, el tercer perfil, menos común, corresponde a la presencia de una inmigración femenina ajena a la reagrupación familiar y más cercana a la realización de un proyecto propio.

En segundo lugar, se constata la existencia de una discriminación que atañe específicamente al colectivo femenino y que se suma, por tanto, a la discriminación la- 
boral y social que afecta en general a la población inmigrante. Así, podemos afirmar que las ocupaciones que se les asignan a las mujeres inmigrantes en el mercado de trabajo español representan una especie de prolongación de las actividades que éstas realizan en la esfera familiar. Esto les lleva a desempeñar mayoritariamente una actividad como el servicio doméstico que está socialmente desvalorizada y sometida a un régimen jurídico especial, claramente desventajoso y discriminatorio para la mujer. Asimismo, este fenómeno hace que la profesión desempeñada en España no guarde en muchos casos relación con su nivel de formación y, por tanto, con sus expectativas laborales. Además, la modalidad de servicio doméstico interno lleva a la mujer inmigrante a una menor disponibilidad de tiempo libre, limitando sus actividades en el campo privado y, por tanto, provocando un importante aislamiento social y una mayor dificultad para adaptarse y acomodarse al nuevo entorno.

Sobre las mujeres inmigrantes recae normalmente el cuidado y la educación de los hijos, tanto si se encuentran en el país de destino como si permanecen en el lugar de origen. Asimismo, los estudios nos indican que la mayoría de las trabajadoras inmigrantes sostienen económicamente a su familia, lo que nos invita a concebir la emigración de la mujer como una estrategia familiar de subsistencia económica y de aumento de los ingresos familiares. Esta situación puede provocar en la mujer una actitud de aceptación pasiva de las condiciones laborales en el país de destino y una falta de predisposición a la reivindicación de sus derechos como trabajadoras.

De otro lado, las mujeres inmigrantes son consideradas como las principales transmisoras y perpetuadoras de su cultura de origen. No es extraño, pues, que experimenten conflictos por la frecuente disparidad en valores, costumbres, normas, etc., exis- tente entre la cultura de su país de origen y la de destino; igualmente, esta percepción de la mujer como piedra angular de la socialización dificulta su distanciamiento de la cultura de origen, frecuentemente de carácter muy conservador y sexista.

Por último, las circunstancias familiares, personales, sociales y laborales que rodean a la mujer inmigrante pueden provocar cierta vulnerabilidad al padecimiento de trastornos psicológicos. Este puede ser el caso de la inmigrante "pasiva" que, dedicada exclusivamente a las tareas del ámbito doméstico, puede vivir un aislamiento cultural y un proceso de adaptación tardío a la sociedad de acogida. Esta situación incrementa su dependencia con respecto al varón para todas las actividades desarrolladas en el campo público. Igualmente, la separación familiar, las dificultades de reagrupación familiar, el estatus de ilegalidad, las duras condiciones laborales, etc., están en la base de la aparición de malestar psicológico en las mujeres trabajadoras inmigrantes.

\section{Características del proceso migratorio femenino desde una perspectiva psicosocial}

Por otra parte, también existen estudios (Gregorio, 1994, 1998; Martínez, 1997; IOE, 1994; Martínez y cols, 1999; Izquierdo, 1994; Marqués, 2000) que reflejan, implícita o explícitamente, la importancia de la perspectiva psicosocial en el análisis de los procesos migratorios, pues ayuda a aclarar algunos aspectos del proyecto de la persona inmigrante que son independientes y ajenos a los factores socioeconómicos, culturales y políticos que se suelen aludir como principales determinantes de los fenómenos migratorios. Hagamos un breve repaso de algunas de las variables psicosociales más relevantes para este campo de estudio. 


\section{Estereotipos de género}

Este proceso psicosocial, que será desarrollado más detenidamente en los epígrafes siguientes, se convierte en una de las claves más importantes para la explicación del fenómeno migratorio femenino al incidir directamente en la percepción que tanto la sociedad como la propia mujer tienen de su proyecto migratorio.

\section{La imitación de patrones conductuales}

Siguiendo a Carmen Gregorio (1994), con esta variable se alude al proceso a través del cual se crea en el país de origen de la mujer una "cultura de la emigración" que ayuda a ésta y a su contexto familiar a percibirla como una estrategia plausible para solucionar sus diversos problemas económicos, socioculturales o políticos.

Bajo este enfoque, la decisión de emigrar puede venir condicionada por la existencia de una historia migratoria familiar y/o comunitaria, pues ésta facilita la percepción de la emigración como la única salida válida para la solución de los diversos problemas que se presentan en su entorno. Igualmente, la presencia en el país de origen de la mujer inmigrante de una tradición migratoria favorece la creación de la infraestructura necesaria (préstamos, documentación, etc.) para llevar a cabo su proyecto migratorio y minimiza, por tanto, las barreras que obstaculizan el salto al país de destino.

\section{Estrategias de inserción laboral}

Independientemente del proceso de etnoestratificación del mercado laboral español al que antes hemos aludido, se pueden identificar otros factores psicosociales que influyen en las diversas formas de inserción laboral que pueden adoptar las mujeres inmigrantes. Así, podemos hablar fundamentalmente de tres: los recursos disponibles (nivel de estudios, cualificación profesional, experiencia laboral previa, etc.) para abrirse camino en el mercado de trabajo, las actitudes que las mujeres posean en torno a los derechos que ellas mismas y los autóctonos tienen en el país de acogida y la creación de redes sociales migratorias.

Con respecto a las actitudes de la mujer inmigrante, se puede hipotetizar que si la nacionalidad del país de destino es percibida como una "idiosincrasia cultural impermeable", las inmigrantes se sentirán intrusas y aceptarán que se les asignen los lugares de trabajo que los autóctonos no desean para ellos. Por el contrario, si perciben el lugar de destino como una sociedad intercultural, éstas reclamarán los mismos derechos y oportunidades que los autóctonos (IOE, 1994; citado por Solé, 1997).

De otro lado, no cabe duda que las redes sociales migratorias juegan un papel fundamental en la obtención de empleo de las personas inmigrantes en el país de destino. Este aspecto adquiere en el caso de la mujer una gran importancia por dos razones, a nuestro juicio, fundamentales: en primer lugar, porque las redes de reclutamiento de mujeres son distintas a las de los hombres; es decir, sus pautas de colocación en los sectores productivos del país de acogida son diferentes a las de los varones (Martín, 1999). En segundo lugar, y muy relacionado con lo anterior, la existencia de redes sociales migratorias favorece la concentración étnica en un determinado sector de actividad (como, por ejemplo, está ocurriendo con el reclutamiento de mujeres dominicanas para la realización del trabajo doméstico en España). Esto es, se produce un proceso de "facilitación étnica" (Light y Bonacich, 1988) donde es la propia mujer inmigrante la que pone en relación la oferta y la demanda de 
trabajo, ofreciendo a las mujeres de su misma nacionalidad la posibilidad de trabajar en ese nicho laboral.

\section{La imagen idílica del emigrante}

$\mathrm{El}$ proyecto migratorio de muchas mujeres tiene como objetivo o finalidad última una mejora en su situación económica actual y/o un ascenso en su posición social. No obstante, y de acuerdo con Gregorio (1994), desde una perspectiva psicosocial, esta variable nos advierte de las expectativas, que suelen tener tanto la mujer inmigrante como sus grupos primarios y de referencia, de conseguir el éxito económico en el país de destino.

Esta asociación entre emigración y éxito puede producir una gran presión para las mujeres ya que, como hemos tenido ocasión de comprobar anteriormente, la realidad en el país de destino dista mucho de esta imagen idealizada. Más concretamente, entre otras consecuencias, puede desencadenar en el lugar de origen, en el contexto familiar de la mujer que emigra, un aumento del nivel de consumo y un abandono de las formas tradicionales de ingresos, supeditando, así, el bienestar familiar al dinero que ésta pueda aportar con su trabajo y demorando en muchas ocasiones el proyecto de retorno.

\section{Comparación social}

El grado de satisfacción y de bienestar de la mujer inmigrante puede estar modulado por los resultados obtenidos de la comparación que ésta realiza entre las condiciones de partida y las condiciones conseguidas en el país de destino, si bien este proceso, a su vez, puede estar moderado por determinadas variables sociodemográficas, tales como el nivel de estudios, estado civil, presencia o no de hijos, edad, etc.
De manera más detallada, esta variable está relacionada con las expectativas que la mujer inmigrante tiene de conseguir ciertos logros en el país de destino. No obstante, dichas expectativas van variando a lo largo del tiempo y van incrementándose a medida que la mujer va consiguiendo sus metas más prioritarias. Por ello, no es de extrañar que su proyecto migratorio y sus necesidades vayan alejándose progresivamente del cumplimiento de las obligaciones que la mujer tiene con su familia y se acerque a la consecución de su propio bienestar en la sociedad de acogida (Gregorio, 1994).

\section{Apoyo social}

Como señalan Martínez y colaboradores (1999), el grado de adaptación de la persona inmigrante al país de acogida, además de estar relacionado con diversas características individuales, tales como los estilos de afrontamiento que posea el individuo, su autoestima o su sentimiento de eficacia o competencia, depende, en gran medida, de la existencia de un sistema de apoyo social. Veamos brevemente su papel como modulador de la satisfacción y el bienestar de la mujer inmigrante.

De acuerdo con los autores mencionados, el sistema de apoyo social en el nuevo contexto está constituido por la existencia de vínculos familiares y sociales (presentes o ausentes) de la red de origen y por la presencia de nuevos vínculos en la sociedad de acogida. Estos recursos sociales juegan un papel importante en el desarrollo del proyecto migratorio: en primer lugar, la decisión de emigrar de la mujer puede venir más determinada por la presencia de lazos familiares en el lugar de destino que por las propias características personales de ésta o por su situación social y económica. En segundo lugar, facilitan la obtención de ayuda y contribuyen a la satisfacción de las 
diversas necesidades materiales, psicológicas y sociales de la mujer inmigrante. En tercer lugar, la existencia de recursos sociales en la sociedad de origen favorece el enfrentamiento a la nueva cultura y el establecimiento de nuevas redes sociales en el lugar de destino.

Así pues, podríamos concluir afirmando que esta variable psicosocial, medida a través del grado en que la mujer inmigrante dispone de recursos sociales procedentes tanto del lugar de origen como de la comunidad de acogida, es de vital importancia en el estudio del proyecto migratorio de las mujeres, pues contribuye a la satisfacción de sus diversas necesidades, y juega un importante papel en su proceso de adaptación a la nueva sociedad y en su bienestar.

\section{Actitudes con respecto a la integra- ción en el país de acogida}

El mayor o menor éxito personal y social conseguido en el país de destino, así como el grado de satisfacción y de bienestar experimentado puede estar mediatizado por las actitudes y las creencias que la mujer inmigrante tenga acerca del concepto de integración. En este sentido, nos podemos encontrar con una gran variedad que puede oscilar desde las que quieren parecerse lo más posible a los autóctonos hasta aquellas inmigrantes que prefieren mantener su propia idiosincrasia, pasando por aquellas otras que opinan que en el término medio está la virtud, estando dispuestas a conseguir la doble nacionalidad, pero manteniendo intactas las costumbres y la cultura de su país de origen (Izquierdo, 1994).

El estudio de esta variable psicosocial es de suma importancia pues nos conduce directamente a reflexionar sobre la bondad de una política migratoria enmarcada y dirigida a la integración total de la población inmigrante, cuando quizás fuera más desea- ble tener en cuenta la diversidad de creencias y actitudes ante la integración y desarrollar una política acorde con dichas opciones.

\section{Investigación de la inmigración femenina desde una perspectiva psicosocial y de género}

En el breve recorrido que hemos efectuado por la literatura que existe sobre el tema de la inmigración, no hemos encontrado demasiados esfuerzos por aunar la perspectiva psicosocial y de género en el análisis del proyecto migratorio femenino, enfoque que, sin duda, nos permitiría intervenir en su entorno microsocial, ayudando a la integración, el bienestar y la satisfacción de la mujer inmigrante.

Por ello, nos gustaría esbozar en las siguientes líneas algunas de las cuestiones generales que se podrían tener en cuenta en las futuras líneas de investigación para lograr una adecuada comprensión del fenómeno migratorio femenino.

\section{Marco teórico para el estudio del}

proyecto migratorio femenino: la Teoría de

la Socialización Diferencial

En primer lugar, creemos que sería conveniente partir de algún marco teórico desde el que se nos permita investigar, explicar e intervenir en el fenómeno de la inmigración femenina desde una perspectiva psicosocial y de género.

Uno de los modelos teóricos más plausibles para conseguir este objetivo es la Teoría de la Socialización Diferencial, ya que consideramos que el proceso de socialización es el punto de partida a partir del cual se prepara a los seres humanos para percibir diferencias entres los sexos y actuar conforme a ello. Asimismo, es en este proceso donde tienen lugar las creencias 
estereotipadas sobre los roles, los rasgos de personalidad, las ocupaciones e, incluso, las características físicas que deben poseer hombres y mujeres. Por último, podemos afirmar que, a través de la socialización, las diferencias reales entre hombres y mujeres se acrecientan y las que no existen se crean para mantener, así, una categorización social que conlleva importantes prejuicios para uno de los dos sexos: la mujer.

En líneas generales, y acogiéndonos a una definición clásica, el concepto de socialización alude al proceso por el que los individuos adquieren el conocimiento, las habilidades y las disposiciones que les capacitan para participar, de forma más o menos efectiva, como miembros pertenecientes a grupos y a una sociedad concreta (Brim y Weeler, 1966).

Por tanto, se trata de un proceso a través del cual el ser humano, influido por sus experiencias y por los agentes sociales más significativos, aprende, adquiere e interioriza, a lo largo de todo el ciclo vital, los elementos socioculturales de su medio ambiente, con el fin de adaptarse al entorno social en cuyo seno debe vivir (Rocher, 1990; Torregrosa y cols., 1989).

Desde este punto de vista, un factor clave en la socialización es la transmisión de roles; es decir, la difusión de un conjunto de expectativas comunes, con carácter normativo, acerca de la conducta de quienes ocupan posiciones específicas en una estructura social (Montero, 1980). En tal sentido, el proceso socializador permite al individuo una adecuada instrucción y entrenamiento en los diversos roles sociales que se verá obligado a poner en funcionamiento a lo largo de su vida.

No obstante, este proceso socializador es diferente según el sexo al que pertenece el individuo puesto que los diversos agentes o agencias de socialización se encargan de crear una identidad de género, de fomentar unas determinadas y diferentes normas de pensar, sentir y actuar entre los sexos. En este contexto es donde adquiere significado el término de "socialización diferencial», entendido como el proceso de asimilación de roles diferenciales entre los sexos y de imposición de diferentes estilos cognitivos, actitudinales y conductuales entre ellos. Asimismo, incluye la transmisión de códigos axiológicos y morales diferenciales entre hombres y mujeres (Blanch, 1992).

Dentro de este marco interpretativo, la socialización adquiere un nuevo significado, pues observamos que, en el contexto de las diferencias sexuales, una de sus funciones básicas es la elaboración de categorías sociales de análisis (en este caso, categorización de género) que permitan a los individuos conocer, comprender y diferenciar los distintos aspectos significativos que constituyen la realidad social.

De igual manera, es en este esquema donde adquieren carta de naturaleza los estereotipos sociales y, sobre todo, los estereotipos de género ya que estos últimos son un claro ejemplo del proceso cognitivo de categorización (Morales y López, 1994) $\mathrm{y}$, como tales, presentan una doble funcionalidad o misión en la aprehensión y comprensión de la realidad social.

De un lado, nos ayudan a diferenciar a los demás en función del género y a tener expectativas y visiones diferentes de las personas según su pertenencia a una u otra categoría de género: proceso de categorización y percepción de los demás en términos de género.

De otro lado, fomentan la creación de una serie de expectativas respecto a nosotros mismos derivadas de nuestra autocategorización dentro de una de las categorías de género: proceso de autopercepción o identidad de género (Moya y Gómez, 1996). 
Asimismo, un aspecto importante que completa el proceso de "estereotípia»social es la evidencia de la existencia de juicios evaluativos asociados a los estereotipos de género. De este modo, los hombres no sólo son vistos como diferentes a las mujeres sino que, también, son considerados superiores a ellas (Lips, 1988).

Dentro de este marco teórico, sería necesario comprobar cómo el proceso de autopercepción del estereotipo de la feminidad puede fomentar en la mujer inmigrante una identidad de género femenino, así como estudiar su influencia en el proyecto migratorio de ésta.

En este sentido, y en líneas generales, se puede hablar de tres ejes estructuradores de la identidad de género femenino (Poal,1993):

En primer lugar, la transmisión de mensajes relacionados con la «invisibilidad», «secundariedad» e «inferioridad» del sexo femenino puede generar en la mujer un sentimiento de inadecuación, una autopercepción de haber nacido en el sexo menos importante y, por tanto, en el sexo «inadecuado» o «incorrecto».

De otro lado, los mensajes de bondad, belleza y discreción, como cualidades inherentes a las mujeres, perfilan una imagen de ésta como un ser suave, tímido, callado, abnegado, cariñoso, amable, poco afirmativo y poco exigente.

Por último, esta ideología sobre el «ser femenino» viene acompañada y reforzada por una realidad cotidiana que:

- Limita las libertades de las mujeres mediante la puesta en práctica de un trato discriminatorio.

- Las impulsa a dedicarse exclusivamente de las tareas domésticas y de educación de los hijos.
- No les estimula ni prepara para participar en el ámbito público, ni para conseguir logros, poder o éxito profesional. Más bien, les pone severos obstáculos a su proyección social y laboral.

Estos acontecimientos que configuran la experiencia femenina, al ser asimilados e interiorizados por la mujer, se convierten en barreras que dificultan o impiden su plena realización personal, social y profesional, favoreciendo un perfil psicosocial de graves consecuencias para el bienestar psicológico de la mujer inmigrante e inadecuado a las demandas de eficacia y eficiencia del ámbito público.

Así pues, resumiendo, podemos aludir a un solo referente o eje estructurador de la identidad femenina: la "domesticidad", definida como un comportamiento o una disposición a prestar atención y a dar respuesta a las necesidades del otro. Significa, por tanto, hacerse cargo, por encima del propio interés, de lo que puedan necesitar o desear los demás. Ello se traduce, entre otros comportamientos, en la necesidad inducida de ser buena esposa y madre, de mantener un buen clima familiar o, bien, en la delegación de aspiraciones laborales y/o formativas en aras del bienestar ajeno (Murillo, 1996).

Asimismo, habría que estudiar cómo los estereotipos de género influyen en el análisis y en la valoración que la sociedad, tanto del país de origen como de destino, hace del proyecto migratorio de la mujer.

Los resultados de los diversos estudios e investigaciones indican que efectivamente el proyecto migratorio de la mujer se realiza en un contexto social imbuido de fuertes creencias estereotipadas que condicionan la actitud de los empleadores del país de destino, padres, esposos e, incluso, de las propias mujeres hacia su papel inmigran- 
te. Desde este punto de vista, las creencias y los estereotipos sobre las diferencias entre géneros se convierten en variables esenciales para analizar, explicar y valorar el papel de la mujer en la emigración. Veamos con algunos ejemplos cómo sucede este fenómeno.

La idea ampliamente difundida de que las mujeres deben responsabilizarse del cuidado de la familia y los hombres deben proveer las necesidades materiales hace que la participación laboral de la mujer inmigrante se entienda como una ayuda a la economía familiar. Así, hemos observado que la emigración de la mujer suele ser percibida por su entorno doméstico como una contribución al mantenimiento económico de la familia, como una ayuda o deber para con la unidad familiar (Gregorio, 1997).

De igual modo, como hemos comprobado anteriormente, las creencias estereotipadas sobre los rasgos de personalidad de hombres y mujeres contribuyen a definir qué actividades $u$ ocupaciones se consideran apropiadas para unos y otras. Esta percepción refuerza la segmentación sexual del mercado de trabajo y favorece la agrupación mayoritaria de las mujeres en el servicio doméstico.

Este hecho tiene, entre otras, las siguientes consecuencias para la mujer inmigrante:

a) Restringe sus estrategias de inserción laboral. Es decir, tanto la elección del país de destino como la obtención de empleo en dicho país vienen muy determinadas por la existencia de redes sociales migratorias, de redes sociales de contacto (familiares o, bien, colonias ya establecidas), por lo que la mayor parte de las mujeres que deciden emigrar acabarán en los mismos empleos que sus compatriotas. b) Puede provocar, sobre todo en las mujeres dedicadas al servicio doméstico como internas, un importante aislamiento social, dificultando su grado de adaptación y acomodación al nuevo entorno.

c) Fomenta el carácter de "invisibilidad" que adquiere la migración femenina, pues la contratación ilegal es la norma general en el sector del trabajo doméstico, impidiéndoles el acceso a un permiso de trabajo, de residencia y a darse de alta en la seguridad social.

\section{Marco específico de la investigación}

De manera más concreta, sería necesario explorar las diversas variables que perfilan el proyecto migratorio de la mujer, dando respuesta en la medida de lo posible a los siguientes objetivos específicos:

1. Estudiar las principales variables que definen la cultura de origen de las mujeres inmigrantes, haciendo especial hincapié en las actitudes, valores e ideologías que su entorno familiar y social sostiene acerca de la relación entre los géneros.

2. Estudiar las actitudes de la propia mujer inmigrante hacia el rol de la mujer en la sociedad y hacia la emigración femenina.

3. Identificar los factores que determinan la decisión de emigrar.

4. Identificar el perfil laboral (motivación laboral, valor atribuido al empleo, aspectos valorados del trabajo, etc.) que presentan las mujeres inmigrantes.

5. Explorar los recursos disponibles (nivel de estudios, cualificación profesional, experiencia laboral previa, estatus legal como extranjera, conocimientos 
del idioma autóctono, etc.) para abrirse camino en el mercado de trabajo, así como su relación con el trabajo real que realizan las mujeres en el país de acogida.

6. Identificar las variables sociodemográficas, psicosociales y de género que modulan y dan variabilidad al proyecto migratorio femenino con la finalidad de evitar la generalización en el análisis del proceso migratorio femenino y de facilitar una intervención acorde con las necesidades y expectativas concretas de estas mujeres.

7. Analizar los factores que facilitan el traslado de las mujeres desde su país de origen al país de acogida, poniendo especial énfasis en las redes de reclutamiento y en los mecanismos o estrategias de inserción laboral de las mujeres inmigrantes en el país de destino.

8. Estudiar cómo las diferentes formas que puede adoptar la inmigración femenina (pasivas, activas con carga familiar y sin ella, solteras y casadas) influyen en el grado y la forma de inserción e integración de la mujer en la sociedad de acogida.

9. Aclarar el papel de las mujeres en los procesos de reagrupación familiar y, más concretamente, su repercusión sobre las actitudes y los valores del grupo familiar con respecto a la emigración.

10. Indagar en las expectativas de las mujeres acerca de su proyecto de retorno, así como averiguar las diversas variables que median en él.

11. Explorar las actitudes de las mujeres hacia el tema de la integración social y cultural en el país de acogida, con el objetivo de averiguar sus preferencias y el grado en que éstas desean y/o están dispuestas a asimilar la cultura del país de destino o, por el contrario, a mantener los hábitos, normas y cultura de su país de origen.

12. Averiguar el grado de satisfacción y de bienestar psicológico, identificando las variables más relevantes que lo modulan.

\section{Conclusiones}

En las páginas precedentes hemos intentado reflexionar sobre la necesidad de profundizar en el estudio de la mujer inmigrante desde una perspectiva psicosocial y de género y ello, desde el marco de la investigación, implica analizar las principales variables psicosociales que definen el proyecto migratorio femenino y ubicar dicho proyecto en un enfoque de género, pues no cabe duda que éste se encuentra fuertemente modulado o mediatizado por una serie de factores que proceden directamente de su condición de mujer.

Creemos que la línea de investigación que se ha ofrecido en estas páginas puede ser de gran relevancia y utilidad social por dos razones, a nuestro juicio, fundamentales: en primer lugar, puede contribuir a la creación de programas de intervención interdisciplinares desde los que se permita dar respuesta al problema de la inmigración en nuestro país de forma más comprehensiva y enriquecedora. En segundo lugar, los resultados obtenidos en el estudio de la inmigración femenina desde la perspectiva que proponemos pueden ayudar a los diversos organismos e instituciones competentes a delimitar las actuaciones hacia la población inmigrante femenina, al ofrecer información detallada de las características que definen el entorno psicosocial de la mujer inmigrante, de las diferencias con respecto a las presentadas por el va- 
rón y de la propia heterogeneidad que probablemente presente el grupo de mujeres inmigrantes en España.

En definitiva, nuestra aportación puede favorecer un mayor y mejor conocimiento sobre la problemática específica de la mujer inmigrante, ayudando así a canalizar las diferentes acciones sociales y a diseñar los programas de intervención autonómi$\cos$ y locales atendiendo a las necesidades específicas de la mujer.

\section{Referencias Bibliográficas}

BLANCH, J.M. (1992). Mujer y mercado de trabajo. Implicaciones teóricas y prácticas de la igualdad de oportunidades y la diferencialidad de orientaciones. UAB. Trabajo de investigación presentado en su oposición a cátedra.

BRIM, O. Y WEELER, S. (1966). Socialization after chilhood: Two essays. New York. Wiley.

Cardona, M.B., 1999. “El trabajo de la inmigrante en España". En "Inmigración y Relaciones Laborales". II Jornadas Andaluzas de Relaciones Laborales. Granada.

Gailly, A. Y Driss, R.B., 1997. "Psicopatologías de los emigrantes en Europa". En Solas, O. y Ugalde, A. (Eds): Inmigración, salud y politicas sociales. Junta de Andalucía y E.A.S.P. Sevilla.

GREGorio, C., 1994. "El grupo inmigrante y su proyecto migratorio: Aspectos psicosociales, culturales y de género". Intervención psicosocial, vol.III, nº7.

Gregorio, C., 1997. "El estudio de las migraciones internacionales desde una perspectiva del género". Migraciones. Instituto Universitario de Estudios sobre Migraciones, $\mathrm{n}^{\mathrm{o}} 1$.
Gregorio, C., 1998. Migración femenina. Su impacto en las relaciones de género. Narcea. Madrid.

Gregorio, C. y Franzé, A., 1999. “Intervención social con población inmigrante: esos otros culturales". Intervención Psicosocial, vol.8, nำ2.

HiTA, C., 1999. “La integración sociolaboral de las inmigrantes: El proyecto mariposa".

En Inmigración y Relaciones Laborales. II Jornadas Andaluzas de Relaciones Laborales. Granada.

IOE. 1994. Marroquins a Catalunya. Barcelona. Generalitat de Catalunya. Institut Catala d'Estudis Mediterranis.

IzQuierdo, A., 1994. "Causas y consecuencias de los movimientos migratorios". En La intervención con inmigrantes desde los servicios sociales comunitarios. Ponencias del curso de formación básica para trabajadores sociales celebrado en Marbella. Junta de Andalucía. Consejería de Trabajo y Asuntos Sociales. Sevilla.

Light, I. y Bonacich, E. 1988. Ethnic Entrepreneurs, Koreans in Los Angeles. Berkeley. University of California.

Lips, H.M. (1988). Sex and gender: An introduction. Mountain View, Ca. Mayfield.

López B. y RamíreZ, A., 1997. “¿España es diferente?. Balance de la inmigración magrebí en España". Migraciones. Instituto Universitario de Estudios sobre Migraciones, $\mathrm{n} ¹$.

López De LeRA, D., 1995. “La migración en España a finales del siglo $X X^{\prime \prime}$. Reis, $\mathrm{n}^{\mathrm{o}}$ 71-72.

MARQués, B., 2000. Las dificultades psicológicas de adaptación de los trabajadores inmigrantes. Confederación de Empresarios de Aragón. 
Martín, E. (dir), 1999. “Procesos migratorios y relaciones interétnicas en Andalucía". Colección Observatorio Permanente de la Inmigración. Ministerio de Trabajo y Asuntos Sociales. Sevilla.

Martínez, M.F., 1997. "Estrés y apoyo social en el proceso migratorio". En Hombrados, M.I.: Estrés y salud. Valencia. Promolibro.

Martínez, M.F., García, M. y Maya, I., 1999. "El papel de los recursos sociales naturales en el proceso migratorio". Intervención psicosocial, vol.8, n²2.

Martínez, R., 1999. “Los nuevos temporeros en Jaén: condiciones sociolaborales de los trabajadores extranjeros". En Inmigración y Relaciones Laborales. II Jornadas Andaluzas de Relaciones Laborales. Granada.

Montero, R (1980). Normas, roles y posiciones sociales. En J.M. Salazar (ed): Psicología Social. México. Trillas.

Morales, J.F. y López, M. (1994). Estereotipos de género y valores. En M. Álvaro (Coord.): Propuesta de un sistema de indicadores sociales de igualdad entre géneros. Madrid. Instituto de la Mujer.

Moya, M. y Gómez, C. (1996). Psicología y género: Un análisis de la profesión. Granada. Servicio de Publicaciones de la Universidad de Granada.
Murillo, S., 1996. El mito de la vida privada. De la entrega al tiempo propio. Madrid. Siglo XXI.

PoAl, G. (1993). Entrar, quedarse, avanzar. Madrid. Siglo XXI.

Prado, C. y cols., 1995. "La mujer inmigrante en España". En Nuevas perspectivas en Antropología Biológica. Diputación Provincial de Granada.

PRADO, C. y cols., 1997. "Ciclo y patrones reproductivos en las mujeres emigrantes en España". En Solas, O. y Ugalde, A. (Eds.) Inmigración, salud y politicas sociales. Junta de Andalucía y E.A.S.P. Sevilla.

RAYA, E.E., 1999. "Notas para el análisis de los problemas y de las políticas de inmigración en España". Intervención Psicosocial, vol.8, nำ.

Rocher, G. (1990). Introducción a la Sociología general. Barcelona. Herder.

Solé, C. (1997). "La irregularidad laboral de la inmigración extracomunitaria". Migraciones. Instituto Universitario de Estudios sobre Migraciones, ํำ1.

TorregrosA, J.R.; Bergere, J. y Alvaro, J.L. (1989). Juventud, trabajo y desempleo: Un análisis psicosociológico. Madrid. Ministerio de Trabajo y Seguridad Social. 
